\title{
Testing the NKT cell hypothesis of human IDDM pathogenesis
}

\author{
Peter T. Lee, ${ }^{1}$ Amy Putnam, ${ }^{2}$ Kamel Benlagha, ${ }^{1}$ Luc Teyton, ${ }^{3}$ Peter A. Gottlieb, ${ }^{2}$ \\ and Albert Bendelac ${ }^{1}$ \\ ${ }^{1}$ Department of Molecular Biology, Princeton University, Princeton, New Jersey, USA \\ ${ }^{2}$ University of Colorado Health Sciences Center, Barbara Davis Center for Childhood Diabetes, Denver, Colorado, USA \\ ${ }^{3}$ Department of Immunology, Scripps Research Institute, La Jolla, California, USA
}

\begin{abstract}
Defects in IL-4-producing CD1d-autoreactive NKT cells have been implicated in numerous Th1-mediated autoimmune diseases, including diabetes, multiple sclerosis, rheumatoid arthritis, lupus, and systemic sclerosis. Particular attention has been focused on autoimmune insulin-dependent diabetes mellitus (IDDM) because nonobese diabetic (NOD) mice and humans with IDDM are both reported to express severe deficiencies in the frequency and Th2 functions of NKT cells. Furthermore, experimental manipulations of the NKT defect in the NOD mouse induced corresponding changes in disease. Taken together, these converging studies suggested a general role of NKT cells in natural protection against destructive autoimmunity. However, in previous reports the identification of NKT cells was based on indirect methods. We have now devised a direct, highly specific CD1d tetramer-based methodology to test whether humans with IDDM have associated NKT cell defects. Surprisingly, although we find marked and stable differences in NKT cells between individuals, our study of IDDM patients and healthy controls, including discordant twin pairs, demonstrates that NKT cell frequency and IL-4 production are conserved during the course of IDDM. These results contradict previous conclusions and refute the hypothesis that NKT cell defects underlie most autoimmune diseases.
\end{abstract}

J. Clin. Invest. 110:793-800 (2002). doi:10.1172/JCI200215832.

\section{Introduction}

NKT cells are a specialized subset of $\mathrm{CD}^{+}$and CD4-CD8- double-negative (DN) $\alpha \beta \mathrm{T}$ cells that are restricted by the non-MHC-encoded antigenpresenting molecule $\mathrm{CD} 1 \mathrm{~d}$ and use a conserved autoreactive TCR, $V \alpha 14-\mathrm{J} \alpha 18 / \mathrm{V} \beta 8$ in mice and $V \alpha 24-J \alpha 18 / V \beta 11$ in humans (see review in ref. 1). Their ability to secrete Th1 and Th2 cytokines as well as chemokines in response to primary stimulation by CD1d-expressing antigen-presenting cells, such as dendritic cells, is thought to underlie their reported regulatory functions in a range of diseases, including cancer and infectious and autoimmune conditions (reviewed in refs. 2, 3).

Selective defects in NKT cell frequency and cytokine secretion have been reported in several autoimmune diseases, suggesting the hypothesis that, like other regulatory T cells $(4,5)$, NKT cells could play a general function of suppressing autoimmunity. For

Received for publication April 30, 2002, and accepted in revised form June 25, 2002

Address correspondence to: Albert Bendelac, Department of Pathology, University of Chicago, 5841 South Maryland Avenue, Chicago, Illinois 60637, USA. Phone: (773) 834-8646; Fax: (773) 702-3701; E-mail: abendelac@bsd.uchicago.edu. Conflict of interest: No conflict of interest has been declared. Nonstandard abbreviations used: double-negative (DN); insulindependent diabetes mellitus (IDDM); nonobese diabetic (NOD); alpha-galactosylceramide ( $\alpha \mathrm{GalCer})$; peripheral blood leukocyte (PBL); allophycocyanin (APC). example, markedly decreased frequencies were reported in humans with insulin-dependent diabetes mellitus (IDDM) $(6,7)$, systemic sclerosis (8), systemic lupus erythematosus $(9,10)$, rheumatoid arthritis $(10,11)$, multiple sclerosis $(12)$, and inflammatory bowel diseases (10). Likewise, NKT cells were reported to be defective in nonobese diabetic (NOD) mice prior to insulitis $(13,14)$ and in diseased NZBXNZW and C57BL/6 $1 \mathrm{pr}^{-/-}$mice (15).

IDDM, a prominent autoimmune disease leading to the destruction of insulin-producing cells in up to $1 \%$ of the U.S. population, serves as a case in point to test the role of NKT cells in autoimmune diseases. In the NOD mouse model, onset of diabetes could be prevented by correction of the NKT cell defect through overexpression or cell transfer $(16,17)$. In addition, aggravation of the NKT defect by introduction of a germline deletion of CD1d led to an exacerbation of disease in NOD mice $(18,19)$. Conversely, specific activation of NKT cells by the synthetic glycolipid antigen $\alpha$-galactosylceramide ( $\alpha$ GalCer) protected against overt diabetes (19-21). Taken together, these observations strongly suggested that NKT cells had a natural suppressor function against anti-islet $\mathrm{T}$ cells and that NKT cell defects had an important, causative role in the NOD disease model. In humans, the proposed link between NKT cells and IDDM rests on original studies of discordant twin pairs, which reported a markedly decreased frequency of NKT cells in the peripheral blood of the diseased twins as well as 
a complete and selective loss of the ability of NKT cell clones to secrete IL-4 $(6,22)$. Because diabetes-free twins had normal NKT cell numbers and functions, the defects reported in diseased twins must have appeared late, prior to, or immediately after disease onset. A recent study examining a different cohort of IDDM patients confirmed the original report and further showed that at-risk "prediabetic" patients had decreased NKT cell numbers and functions as well, suggesting that the NKT cell defect appeared prior to overt disease (7). Intriguingly, a concomitant defect in $\mathrm{CD} 4{ }^{+} \mathrm{CD} 25^{+}$"regulatory" $\mathrm{T}$ cells was reported, suggesting a generalized failure of suppressor mechanisms controlling autoimmunity.

These studies, however, lacked a direct, specific method for enumerating and functionally testing NKT cells. The original studies of discordant twins used flow cytometry to identify $\mathrm{CD}^{-} \mathrm{CD} 8^{-} \mathrm{V} \alpha 24^{+}$ cells in fresh peripheral blood leukocytes (PBLs) and Vo24 sequence analysis of in vitro-derived $\mathrm{T}$ cell clones to score the invariant canonical sequences. Thus, they missed the $\mathrm{CD} 4^{+}$component of NKT cells, which we and others recently showed to be the major source of IL-4 $(23,24)$, and they provided calculated rather than directly enumerated NKT cell frequencies. The more recent study used a combination of $V \alpha 24$ and V $\beta 11$ mAb's that did not differentiate CD1drestricted NKT cells bearing the canonical Vo24-J $\alpha 18$ chain from conventional $\mathrm{T}$ cells. Furthermore, because human NKT cells are rare, particularly in the blood where their frequency is often lower than $0.1 \%$ in healthy subjects, these studies raised the technical issue of the reliability of flow cytometry to identify very rare cell subsets.

We have devised a method that specifically and reproducibly identifies one canonical NKT cell among 1 million (0.0001\%) PBLs. It is based on the combined use of CD1d tetramers loaded with $\alpha$ GalCer and the anti-V $224 \mathrm{mAb}$ (23). Using this method, we have reexamined the notion that IDDM patients exhibit NKT cell defects. First, we observed that NKT cell numbers in healthy subjects were considerably lower than previously estimated and, while remarkably stable over time in any given individual, varied considerably a

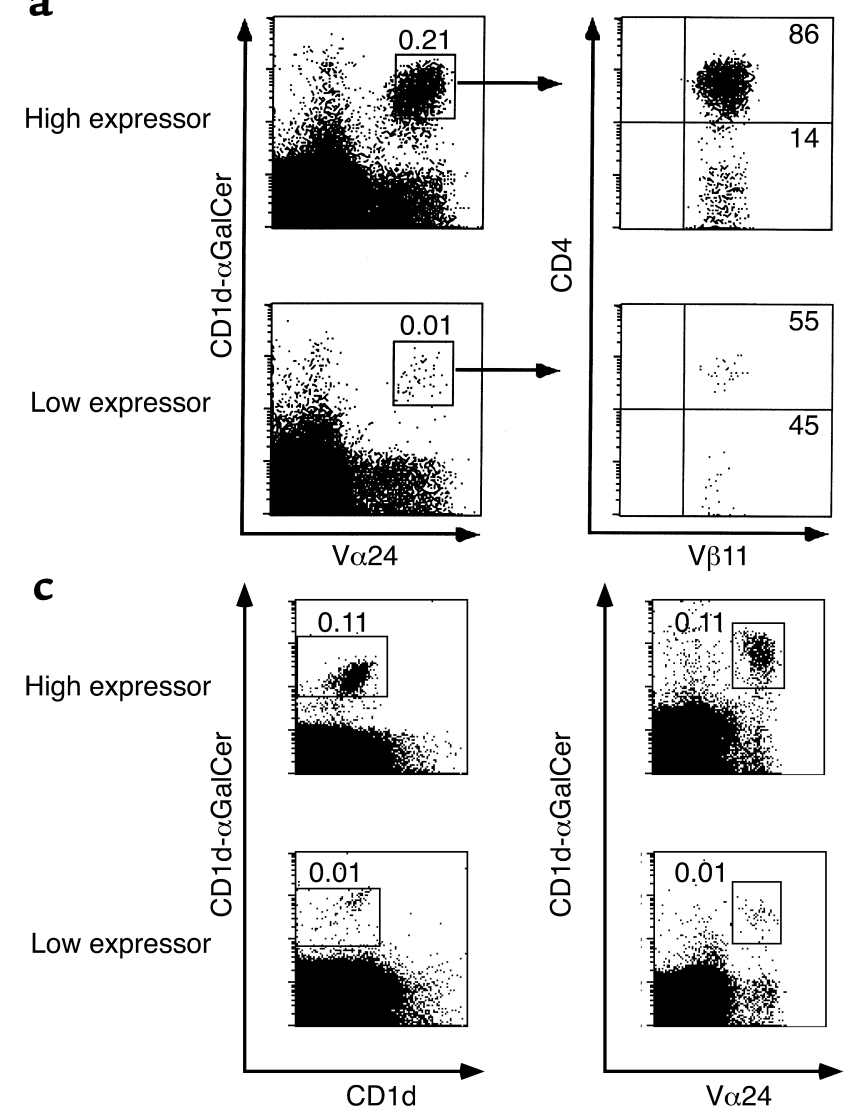

b

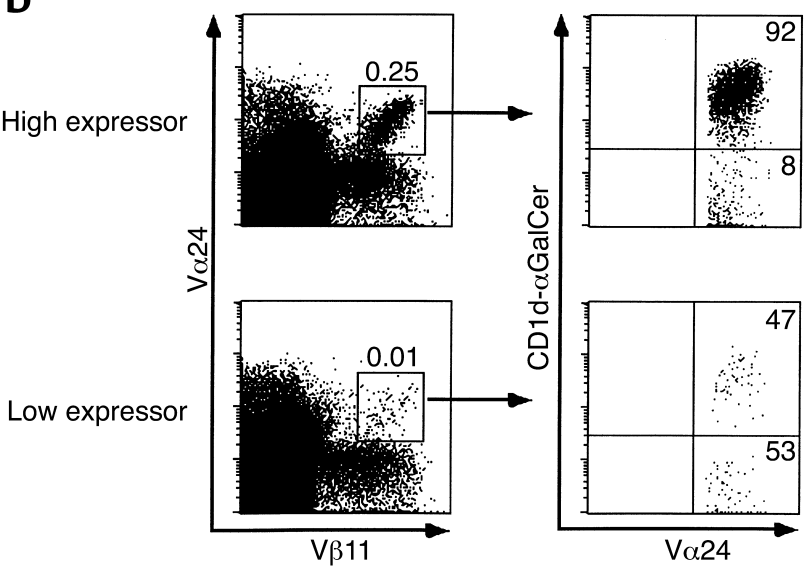

Figure 1

Specific identification of human NKT cells in PBLs. (a) NKT cells in PBLs were identified by double staining with CD1d- $\alpha$ GalCer tetramers followed by anti-V $\alpha 24 \mathrm{mAb}$ (left panels). The CD4 distribution and V $\beta 11$ usage among these cells were identified in the same staining (right panels). (b) V $\alpha 24 / V \beta 11$ double-positive PBLs (left panels) were stained with CD1d- $\alpha$ GalCer tetramers (right panels). Numbers above the boxes represent percentages of cells among total PBLs, whereas numbers in the quadrants represent percentages among boxed cells. Representative FACS plots of donors expressing high or low frequency of NKT cells are shown. (c) Double staining with CD1d- $\alpha$ GalCer and empty CD1d (left panels) identified the same percentage of NKT cells as double staining with CD1d- $\alpha$ GalCer and V $\alpha 24$ (right panels). 


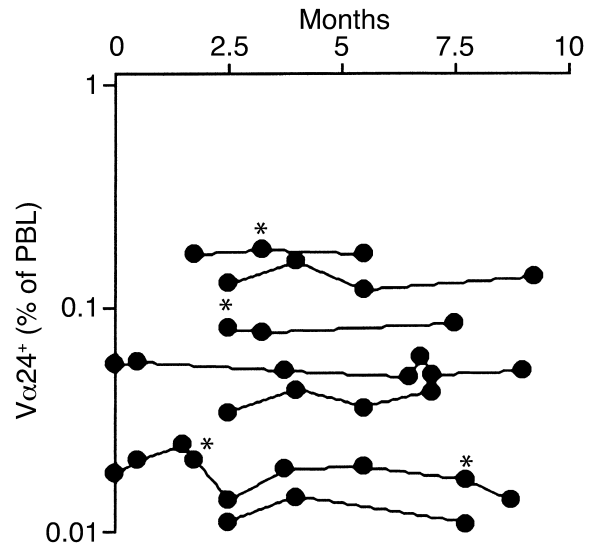

between individuals. Second, we found that NKT cell numbers and cytokine-secreting functions were not significantly altered in a large group of diabetic and prediabetic patients as compared with controls, including discordant twins.

These results directly challenge the notion that autoimmune diabetes in humans is caused or aggravated by an NKT cell defect and suggest that a general reassessment of the role of NKT cells in other diseases is warranted, based on the new identification methods. More generally, this study calls into question the prevailing notion that most autoimmune diseases are caused or aggravated by a general breakdown of suppressor mechanisms such as those exerted by NKT cells.

\section{Methods}

Subjects and cells. The studies of NKT cells were carried out on total PBLs isolated from peripheral blood of donors by using Ficoll gradient according to manufacturer's protocol (Amersham Pharmacia Biotech Europe $\mathrm{GmbH}$, Uppsala, Sweden). Samples were then frozen and stored at $-80^{\circ} \mathrm{C}$ for $1-3$ weeks prior to batch analysis. Each batch consisted of 10-20 samples, including samples from control subjects and diabetic patients. We verified on multiple occasions that both the frequency and function of NKT cells were conserved between fresh and frozen samples (data not shown). All samples were treated and analyzed without prior knowledge of the disease status.

All IDDM subjects were diagnosed at the Barbara Davis Center for Childhood Diabetes, University of Colorado Health Sciences Center, Denver, Colorado, USA,

\section{Figure 3}

Conserved expression of NKT cells in individuals with IDDM. (a) Summary plot showing individual results in controls, subdivided in ageand DQ-matched individuals (cohort 1) and healthy volunteers (cohort 2), at-risk individuals (separated into low [L], medium [M], and high $[\mathrm{H}]$ risk), and IDDM new onset (NO) and long-standing (LS) individuals. (b) Representative FACS plots of healthy or IDDM individuals expressing high or low frequency of NKT cells in PBLs. (c) Summary plot of the relative proportion of $\mathrm{CD} 4^{+}$cells among V $\alpha 24$ NKT cells in control, at-risk, and diabetic individuals.

\section{Figure 2}

Stability of NKT cell frequency in PBLs. V $\alpha 24^{+}$CD1d- $\alpha G C^{+}$NKT cell frequencies were repeatedly measured over a period of 10 months in seven individual donors. Asterisks denote concomitant seasonal rhinovirus or influenza-like infection.

and were extensively studied for serum autoantibody levels and HLA DQ/DR allotypes, as described (25). IDDM was classified as new onset (less than 1 month after diagnosis) or long standing (up to 10 years after diagnosis). At-risk patients, identified at the Barbara Davis Center, were stratified according to high risk if they had three anti-islet autoantibodies (against GAD65, ICA512, insulin) or at least one autoantibody and impaired firstphase insulin release during intravenous glucose tolerance test, medium risk if they had two autoantibodies, and low risk if one autoantibody was detected. Control subjects included age- and HLA-DQ-matched individuals with no family history of diabetes (group 1, identified at the Barbara Davis Center) and healthy volunteers (group 2, from Princeton University). Most controls and IDDM were more than 20 years old, whereas most at-risk individuals were between 10 and 20 years old.

a

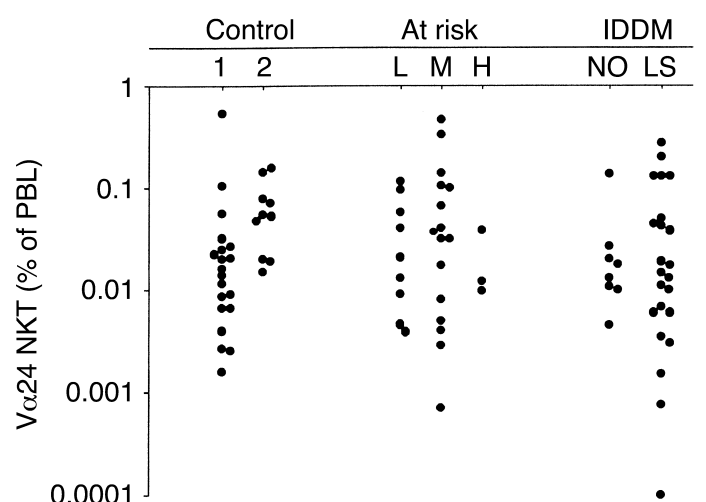

b

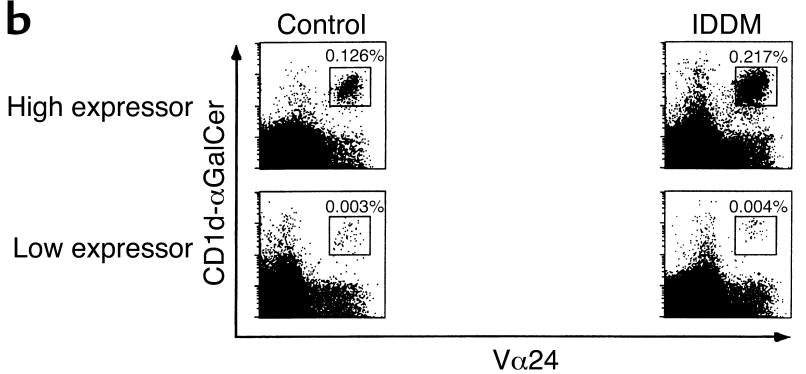

C

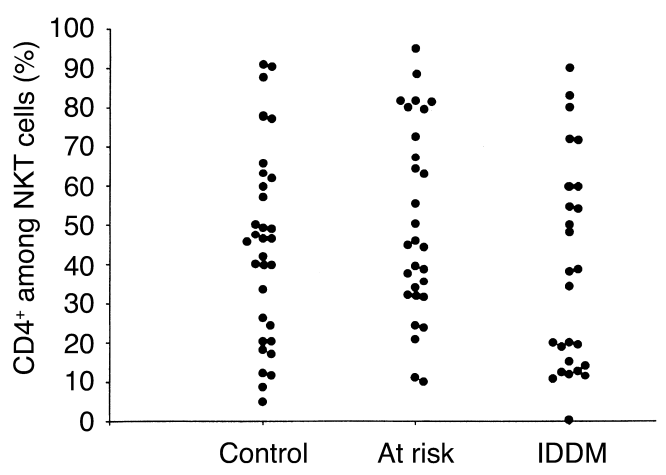


Table 1

NKT cell frequency amongst PBLs of control, at-risk, and IDDM patients according to DQ8 expression

\begin{tabular}{lcc}
\hline \multicolumn{2}{c}{ Vo24 NKT (\% of PBLs) } \\
& $\mathrm{DQ8}^{+}$ & $\mathrm{DQ8}^{-}$ \\
$\begin{array}{l}\text { Control } \\
\text { (group 1) }\end{array}$ & $0.07 \pm 0.09(n=4)$ & $0.05 \pm 0.13(n=17)$ \\
At-risk & $0.08 \pm 0.12(n=17)$ & $0.04 \pm 0.04(n=10)$ \\
IDDM & $0.03 \pm 0.04(n=14)$ & $0.04 \pm 0.07(n=18)$
\end{tabular}

$A b$ 's, CD1d tetramers, and flow cytometric analysis. Fluorochrome or biotin conjugates of Ab's against $V \alpha 24$, Vß11, CD4 (Beckman Coulter Inc., Brea, California, USA), IL-4, and IFN- $\gamma$ (BD Biosciences, San Jose, California, USA) were used. Mouse CD1d- $\alpha$ GalCer tetramers were produced as described (26), and staining was as follows. Cells were incubated with $1 \mu \mathrm{g} / \mathrm{ml}$ unlabeled streptavidin (Pierce Chemical Co., Rockford, Illinois, USA) for 15 minutes at room temperature, followed by incubation with CD1d- $\alpha$ GalCer tetramers for 1 hour at room temperature. Other mAb's such as $\mathrm{CD} 4, \mathrm{~V} \alpha 24$, and $\mathrm{V} \beta 11$ were then added for a further 30-minute incubation on ice. We used a 1:10 dilution of PE-conjugated anti-V $\alpha 24 \mathrm{Ab}$ that did not displace the CD1d- $\alpha$ GalCer tetramers. Cells were then washed with staining buffer (PBS, $0.1 \%$ BSA, and $0.01 \%$ sodium azide; Sigma-Aldrich, St. Louis, Missouri, USA) and analyzed by flow cytometry using FACSort and CellQuest software (Becton Dickinson Immunocytometry Systems, San Jose, California, USA). For studies involving intracellular cytokines, cells were first stained with tetramers, then permeabilized with Cytofix/Cytoperm (BD Biosciences) and washed with Perm/Wash buffer (BD Biosciences). Anticytokine mAbs were then added for 30 minutes before two washes with Perm/Wash buffer. Because the frequency of NKT cells is low, at least $10^{6}$ cells were recorded and analyzed for each staining combination. The frequency of NKT cells was estimated as the average of multiple determinations over a sample size totaling 3-6 million PBLs per individual.

Stimulation of cytokine production. PBLs were cultured for 12 hours at a concentration of $5 \times 10^{6}$ cells $/ \mathrm{ml}$ in 24-well plates in RPMI supplemented with $10 \%$ FCS (Biofluids, Rockville, Maryland, USA) in the presence of $1 \mathrm{ng} / \mathrm{ml}$ of PMA, $1 \mu \mathrm{M}$ of ionomycin, and $5 \mu \mathrm{g} / \mathrm{ml}$ of Brefeldin A (all from Sigma-Aldrich).

\section{Results}

Specific identification of rare CD1d-restricted V $\alpha 24-J \alpha 18$ $N K T$ cells in peripheral blood. We reported recently that specific identification of rare CD1d-restricted NKT cells in the peripheral blood of healthy humans could be achieved by the combined use of $\alpha$ GalCer-loaded mouse CD1d tetramers and the anti-V $\alpha 24 \mathrm{mAb}(23)$. This is shown in Figure 1a, where $100 \%$ of $\mathrm{V} \alpha 24^{+} / \mathrm{CD} 1 \mathrm{~d}-\alpha \mathrm{GalCer}^{+}$double-positive cells were demonstrated to coexpress $V \beta 11$, the characteristic TCR- $\beta$ chain used by human NKT cells. This was found to be true in all samples, regardless of very high $(0.21 \%)$ or very low $(0.01 \%)$ frequency of NKT cells. In contrast, Figure $1 \mathrm{~b}$ shows that the combination of V $\alpha 24$ and V $\beta 11 \mathrm{mAbs}$ was unreliable. Whereas it identified CD1d-restricted NKT cells with some accuracy in some subjects, particularly those with relatively high NKT cell frequency (upper panels, 92\% of $\mathrm{V} \alpha 24^{+} \mathrm{V} \beta 11^{+}$were $\left.\mathrm{CD} 1 \mathrm{~d}-\alpha \mathrm{GalCer}{ }^{+}\right)$, it was grossly inaccurate, particularly among subjects with lower NKT cell frequency, as shown by the large proportion of CD1d- $\alpha$ GalCer cells (lower panel, $45 \%$ of gated cells were tetramer negative). In fact, the majority of $\mathrm{V} \alpha 24^{+} \mathrm{V} \beta 11^{+}$were CD1d- $\alpha \mathrm{GalCer}-$ in up to $20 \%$ of subjects examined (data not shown). In control experiments, we showed that prior incubation with CD1d$\alpha$ GalCer tetramers did not prevent anti-V $\alpha 24 \mathrm{mAb}$ binding in any fraction of Vo24 NKT cells and, conversely, anti-V $\alpha 24$ did not displace the tetramers at the concentration used, because the combination of allophycocyanin-conjugated (APC-conjugated) CD1d- $\alpha$ GalCer and CyChrome-conjugated unloaded CD1d tetramers stained the same number of PBLs as the combination of APC-conjugated $\mathrm{CD} 1 \mathrm{~d}-\alpha \mathrm{GalCer}$ and PE-conjugated anti-V $\alpha 24$ (Figure 1c). Therefore, double staining with $C D 1 d-\alpha$ GalCer

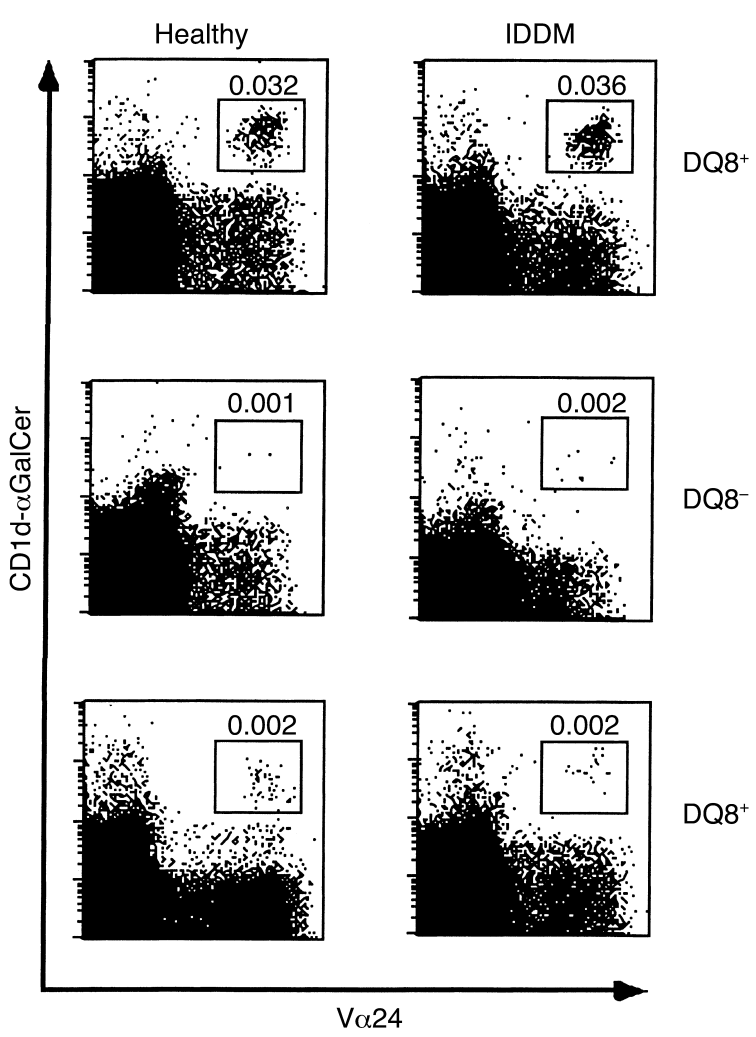

Figure 4

Frequency of NKT cells in twins with discordant IDDM status. Healthy twins did not show evidence for circulating anti-islet $A b$ 's or altered intravenous glucose tolerance test. One of three twin pairs expressed DQ8, as shown. 
and anti-V $\alpha 24 \mathrm{mAb}$ appeared to be a reliable method that accurately and unambiguously identified CD1drestricted V $\alpha 24$ NKT cells in fresh PBLs.

Stability of V 24 NKT cells in peripheral blood. To determine the significance of a single determination of NKT cell frequency in the blood, we ascertained the stability of NKT cell frequency in PBLs over time. Thus, we followed a cohort of seven healthy subjects, repeatedly measuring their NKT cell levels over a period of 10 months. As shown in Figure 2, the frequency of NKT cells was remarkably stable, even at times of seasonal rhinovirus or influenza-like infections (denoted by asterisks).

Conserved frequency of Vo24 NKT cells in IDDM subjects. To reexamine the claim that the frequency of NKT cells in peripheral blood drastically decreases prior to IDDM onset, we studied a cohort of 101 subjects divided into three groups comprising control, at-risk, and IDDM patients (Figure 3). The control group included a "cohort 1 " of individuals with no family history of diabetes that were age- and HLA-DQ-matched with IDDM patients (Table 1) and a "cohort 2" of healthy volunteers. The at-risk group was divided into low (L), medium (M), and high $(\mathrm{H})$ risk, and the IDDM group was divided into new onset (NO) and long-standing (LS), as described in Methods. As shown in Figure 3a, all three groups displayed a broad spectrum of NKT cell frequencies spanning a 100 -fold range. However, there were no significant differences between any of the at-risk or IDDM groups and the control groups. There were no significant differences either between $\mathrm{DQ}^{+}$and DQ8 ${ }^{-}$individuals (Table 1). Representative FACS plots are shown in Figure $3 \mathrm{~b}$ to illustrate that subjects with very low or very high frequency of NKT cells could be found in both the control and the IDDM groups. Finally, to overcome potential unforeseen problems associated with the great genetic heterogeneity between human subjects, we studied three sets of twins who were discordant for diabetes. As shown in Figure 4, the NKT cell frequency was remarkably similar within twin pairs, arguing that it is mainly determined by genetic factors and is not altered by diabetes.

Vo24 NKT cells from diabetic patients are able to secrete IL-4 ex vivo. Because NKT cell-mediated protection against diabetes in NOD mice is believed to depend on IL-4 (16, 17) and humans with IDDM were reported to lack IL-4producing NKT cells $(6,22)$, we examined cytokine production ex vivo by intracellular staining of fresh PBLs treated with ionomycin and PMA. As shown in Figure 5 and reported previously by us and others $(23,24)$, the
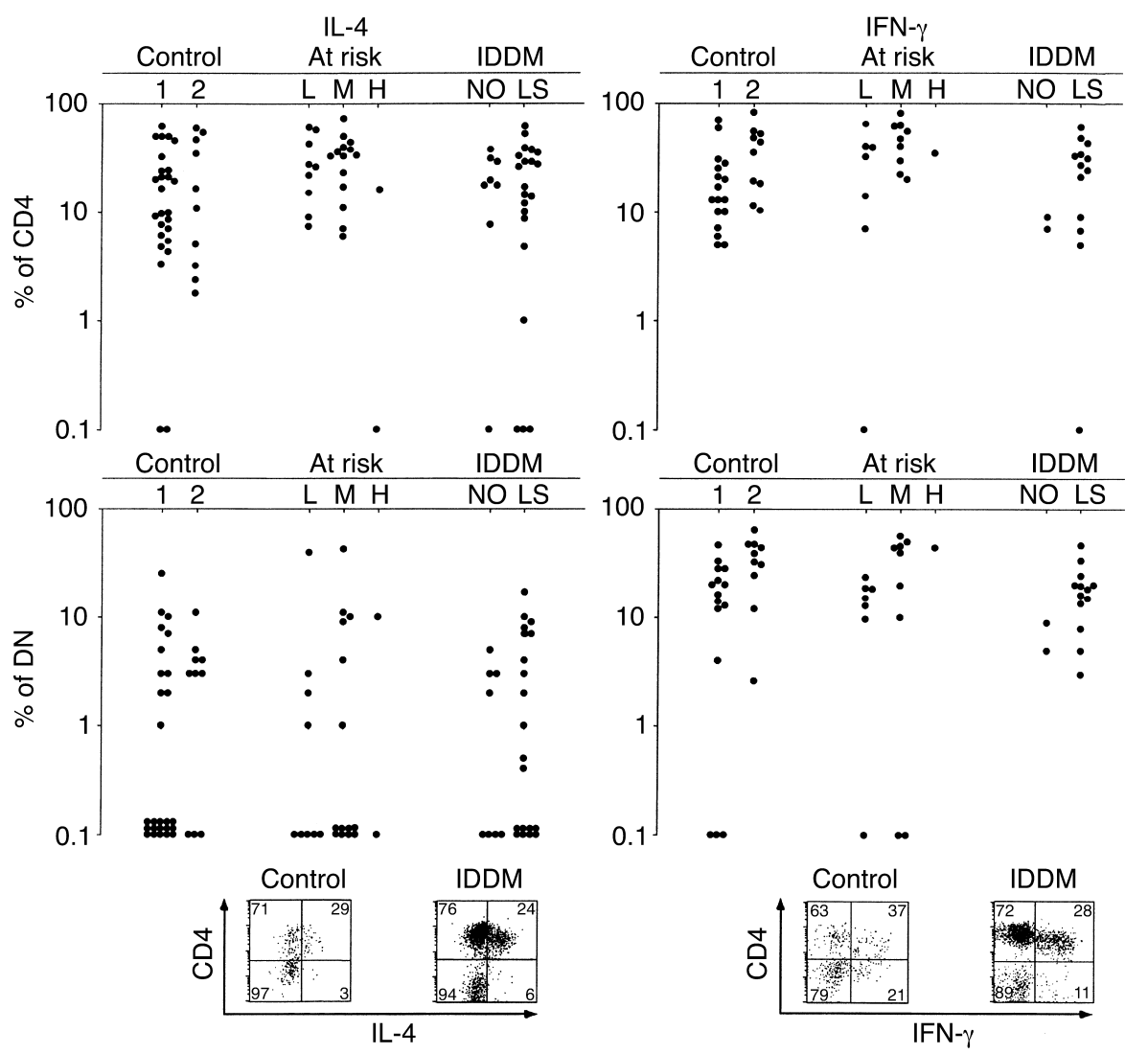

Figure 5

Cytokine production by fresh NKT cells. Summary plots show frequencies of IL-4 (left panels) and IFN- $\gamma$ (right panels) producers among CD4 NKT cells (upper panels) and DN NKT cells (middle panels) in control, at-risk, and IDDM individuals. Representative FACS staining of IL-4 and IFN- $\gamma$ are shown in control and IDDM individuals (bottom panels). 

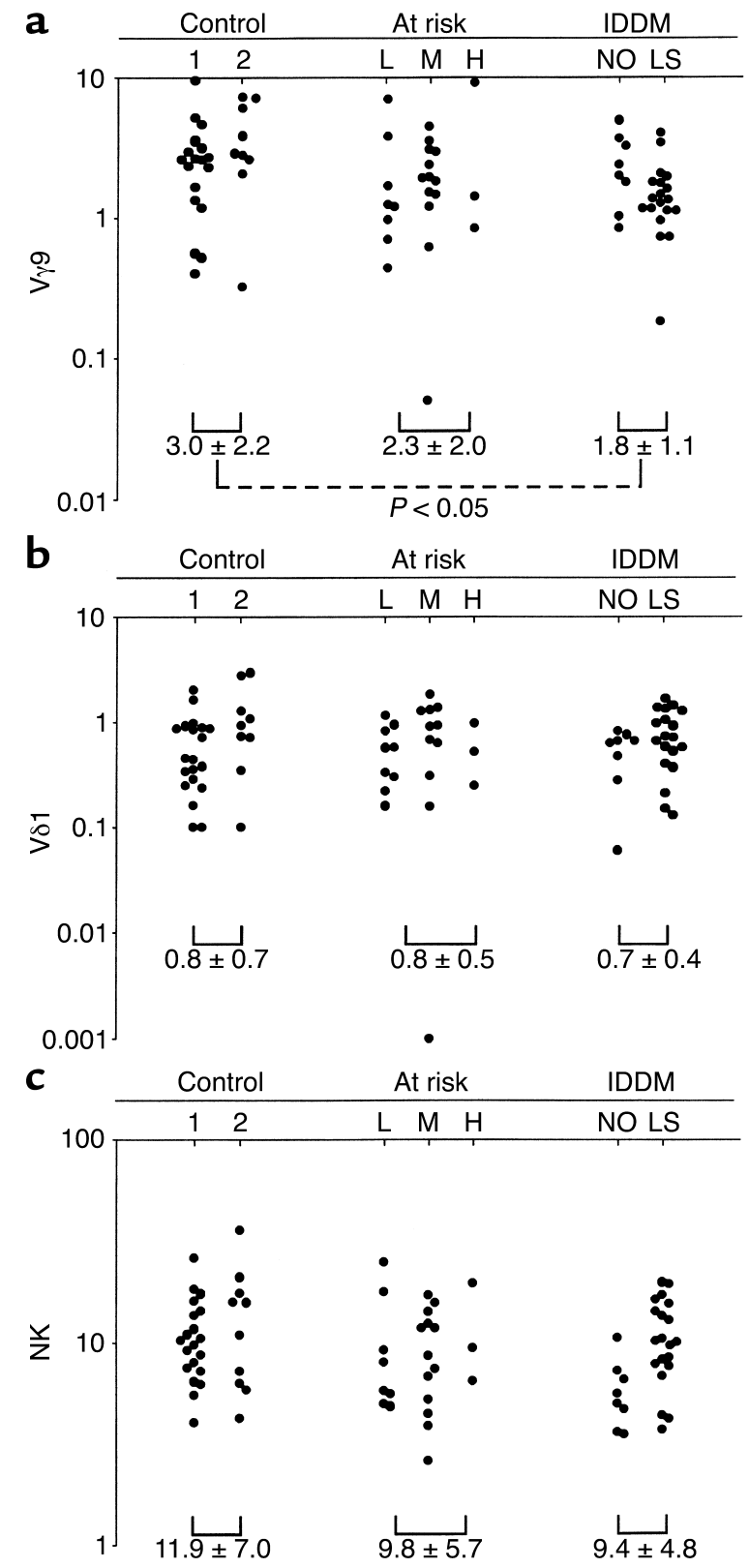

Figure 6

Frequency of different populations of innate-like lymphocytes in PBLs. The $\gamma \delta$ T cells expressing (a) $\vee \gamma 9$ or $(\mathbf{b}) \mathrm{V} \delta 1$ and (c) NK cells were measured in control, at-risk, and IDDM individuals. Mean \pm SD in each group were compared using Student $t$ test.

CD4 subset of V $\alpha 24$ NKT cells is the primary producer of IL-4. Again, in contrast with previous claims, we found no significant differences between diabetic and control individuals. The patterns of IFN- $\gamma$ secretion by V $\alpha 24$ NKT cells were also identical in control, at-risk, and IDDM groups. Similarly, twins discordant for IDDM did not display significant differences in their cytokine profile. For example, the twin pair expressing the highest frequency of NKT cells $(0.03 \%$, see upper panels in Figure 4$)$ also had a similar frequency of IL-4 producers $(10.8 \%$ for the healthy and $10.3 \%$ for the diseased twin).
Since the CD4 subset of NKT cells appears to be primarily responsible for the secretion of protective IL-4 (Figure 5 and refs. 23, 24), we asked whether IDDM subjects had a biased CD4/DN ratio in their Vo24 NKT cell subset. We have found that this was not the case (Figure 3c).

Other innate-like lymphocyte subsets. We have suggested that V $\alpha 24$ NKT cells, like the $\gamma \delta$ T cell and B-1 B cell subsets, belonged to a group of innate-like lymphocytes that express conserved, germline encoded, self-reactive specificities $(27,28)$. These innate-like lymphocytes have specialized functions adapted to their tissue of residence, apparently reacting to conditions of stress, cell injury, and tissue damage, thus more resembling NK cells than conventional T and B cells. We therefore concomitantly measured the frequency of some of these lymphocyte subsets, including $V \gamma \gamma$ and $V \delta 1 \mathrm{~T}$ cells and NK cells in PBLs of IDDM and control subjects. NK cells $\left(\mathrm{CD} 3^{-} \mathrm{CD} 56^{+}\right)$and $\mathrm{V} \delta 1^{+}$ $\mathrm{T}$ cells appeared unaltered in IDDM patients. Interestingly, however, we found a modest, but significant $(P<0.05), 40 \%$ decrease on average of $\mathrm{V} \gamma 9^{+} \mathrm{T}$ cells in IDDM subjects (Figure 6).

\section{Discussion}

The present report is, to our knowledge, the first blind, large-scale study comparing V $\alpha 24$ NKT cells in IDDM patients, pre-IDDM patients, and controls using a direct, specific method for NKT cell identification in fresh PBLs. This method, based on the combined use of mouse CD1d- $\alpha$ GalCer tetramers and anti-V $\alpha 24 \mathrm{mAb}$, can accurately detect one V $\alpha 24$ NKT cell in 1 million fresh PBLs and allows for direct assessment of cytokine profile by intracellular FACS staining. Furthermore, the use of mouse rather than human CD1d tetramers in our study is unlikely to significantly alter the frequency estimates of this rare population, because we and others have shown greater than $95 \%$ cross-reactivity $(26,29)$. Overall, the results collected from over 100 subjects, including three pairs of discordant twins, unambiguously demonstrated the absence of IDDM-associated defect in the frequency of NKT cells in peripheral blood or in their ability to secrete IL- 4 and IFN- $\gamma$ upon primary stimulation ex vivo. This study involved the analysis of 1-6 million PBLs in each blood sample to acquire sufficient numbers of V $\alpha 24$ NKT cells to accurately determine frequency, phenotype, and function. Repeated examination of different samples obtained at different times from either healthy or IDDM subjects showed the remarkable stability of NKT cell frequency in peripheral blood over a follow-up period of up to 10 months and further confirmed the precision and reliability of the method.

Our results conflict with previous studies from two independent groups, predating the availability of CD1d tetramer. One study by Wilson et al. examined seven sets of discordant twins $(6,22)$. This study examined the DN subset of V $\alpha 24$ NKT cells and 
deduced, rather than directly measured, the frequency of DN NKT cells from the frequency of V $\alpha 24-J \alpha 18$ rearrangements among $\mathrm{T}$ cell clones derived after in vitro expansion of DN V $224^{+}$PBLs. Furthermore, cytokine properties were examined at the level of DN $T$ cell clones. We and others $(23,24)$ have shown that DN NKT cells did not secrete Th2 cytokines upon primary stimulation, although we have found that IL-4 could be induced in this subset after repeated stimulations in vitro (data not shown). Thus, IL-4 secretion by in vitro expanded DN T cell clones is not an accurate reflection of the cytokine status of NKT cells in vivo. Therefore, it is possible that the reliance on in vitro-derived DN T cell clones may have confounded both the determination of frequency and cytokine status of fresh NKT cells.

Another study (7) used the combination of anti$\mathrm{V} \alpha 24$ and anti-V $\beta 11 \mathrm{mAbs}$, and in some cases a new $\mathrm{mAb}$ reported to be specific for the canonical Vo24Ja18 junction, to enumerate and test cytokine profiles of fresh Vo24 NKT cells in a large group of IDDM, pre-IDDM, and control subjects. Although we have shown that the combination of anti-V $\alpha 24$ and anti-V $\beta 11 \mathrm{Ab}$ 's does not accurately identify $\mathrm{V} \alpha 24$ NKT cells, we nevertheless did not find significant differences in V $\alpha 24 / \mathrm{V} \beta 11^{+}$cells between IDDM and control groups in our study (data not shown). However, it appears that the main difference between the two studies resides in the control groups. Kukreja et al. (7) found very high frequencies of NKT cells, averaging $0.5 \%$ of PBLs in healthy individuals. This is a frequency that we and others have found only in exceptional cases. For example, studies by Davodeau et al. (30) and Prussin and Foster (31) found $\mathrm{V} \alpha 24 / \mathrm{V} \beta 11^{+}$cell frequencies to be generally less than $0.2 \%$ of PBLs. Indeed, Prussin and Foster indicated that the majority of the donors they screened had undetectable numbers of V $\alpha 24$ NKT cells, below $0.1 \%$ (31). In this context, the finding by Kukreja et al. (7) that NKT cells in IDDM and pre-IDDM exhibited an overall decrease in both frequency and cytokines could reflect instead a bias in the control group toward high NKT expressors.

A decisive argument in support of our conclusion that IDDM is not associated with alterations of $V \alpha 24$ NKT cells is the general agreement of our results both in outbred subjects and in identical twins discordant for diabetes. Overall, the data indicate that V $\alpha 24$ NKT cell levels in humans are probably determined by genetic factors (as in mice) and are not altered by the autoimmune process leading to IDDM. Our results do not exclude the possibility that more subtle NKT cell changes could be associated with or even contribute to pathogenesis. In addition, systematic differences may exist between the cohorts of IDDM or control subjects seen in different centers. Although we think that this is unlikely, future studies in other diabetes referral centers using our CD1d tetramer/V $\alpha 24 \mathrm{mAb}$-based method should clarify this issue.
Our results invite a reappraisal of the evidence presented so far in support of the possible function of NKT cells in IDDM. In the case of NOD mice, there is a consensus over the existence of a partial NKT cell defect (which we have confirmed using CD1d- $\alpha$ GalCer tetramers, data not shown), but genetic evidence based on the introduction of the germline deletion of CD1d in the NOD strain is controversial. Two studies reported an increase in diabetes incidence in CD1d-deficient NOD mice $(18,19)$, whereas another one did not find significant changes (21). Our unpublished results support the latter conclusion. The discrepancy between these studies may be related to genetic heterogeneity, which plays a major role in the expression of complex diseases such as diabetes, in backcrossed mouse strains. Thus, formal genetic proof for the existence or absence of a CD1d effect awaits transgenic complementation of the CD1d mutation or direct inactivation of the CD1d gene in NOD. On the other hand, cell transfer and transgenic expression experiments support a suppressor function of NKT cells against diabetes (16, 17), although their physiological relevance needs to be addressed further.

The present study also demonstrates the need for further studies of autoimmune diseases other than IDDM where putative NKT cell defects have been reported, including systemic sclerosis (8), systemic lupus erythematosus $(9,10)$, rheumatoid arthritis $(10,11)$, multiple sclerosis $(12)$, and inflammatory bowel diseases (10). Using the CD1d- $\alpha$ GalCer tetramer/V $\alpha 24 \mathrm{mAb}$ method described here to reliably identify Vo24 NKT cells will be necessary to reexamine these claims.

In summary, our study demonstrated the absence of Vo24 NKT cell defects in human subjects with IDDM or pre-IDDM, challenging the current prevailing idea that NKT cell defects are invariably associated with autoimmunity.

\section{Acknowledgments}

We thank George Eisenbarth for help with the experimental design and for reviewing the manuscript. This work was supported by grants from the Juvenile Diabetes Foundation International and the NIH (A. Bendelac, P. Gottlieb, and L. Teyton) and by fellowships from the Juvenile Diabetes Foundation International (P. Lee) and the Leukemia and Lymphoma Society of America (K. Benlagha).

\footnotetext{
1. Bendelac, A., Rivera, M.N., Park, S.-H., and Roark, J.H. 1997. Mouse CD1specific NK1 T cells. Development, specificity, and function. Annu. Rev. Immunol. 15:535-562.

2. Smyth, M.J., and Godfrey, D.I. 2001. NKT cells and tumor immunity-a double edged sword. Nat. Immunol. 1:459-460.

3. Park, S.H., and Bendelac, A. 2000. CD1-restricted T-cell responses and microbial infection. Nature. 406:788-792.

4. Shevach, E.M. 2000. Regulatory T cells in autoimmmunity. Annu. Rev. Immunol. 18:423-449.

5. Gonzalez, A., Andre-Schmutz, I., Carnaud, C., Mathis, D., and Benoist, C. 2001. Damage control, rather than unresponsiveness, effected by protective DX5+ $\mathrm{T}$ cells in autoimmune diabetes. Nat. Immunol. 12:1117-1125.
} 
6. Wilson, S.B., et al. 1998. Extreme Th1 bias of invariant Va24JaQ T cells in type I diabetes. Nature. 391:177-181.

7. Kukreja, A., et al. 2002. Multiple immuno-regulatory defects in type-1 diabetes. J. Clin. Invest. 109:131-140. doi:10.1172/JCI200213605.

8. Sumida, T., et al. 1995. Selective reduction of T cells bearing invariant V alpha $24 \mathrm{~J}$ alpha $\mathrm{Q}$ antigen receptor in patients with systemic sclerosis. J. Exp. Med. 182:1163-1168

9. Oishi, Y., et al. 2001. Selective reduction and recovery of invariant Valpha24JalphaQ T cell receptor T cells in correlation with disease activity in patients with systemic lupus erythematosus. J. Rheumatol. 28:275-283.

10. van der Vliet, H.J., et al. 2001. Circulating V(alpha24+) Vbeta11+ NKT cell numbers are decreased in a wide variety of diseases that are characterized by autoreactive tissue damage. Clin. Immunol. 100:144-148.

11. Yanagihara, Y., Shiozawa, K., Takai, M., Kyogoku, M., and Shiozawa, S. 1999. Natural killer (NK) T cells are significantly decreased in the peripheral blood of patients with rheumatoid arthritis (RA). Clin. Exp. Immunol. 118:131-136.

12. Illes, Z., et al. 2000. Differential expression of NK T cell V alpha 24J alpha Q invariant TCR chain in the lesions of multiple sclerosis and chronic inflammatory demyelinating polyneuropathy. J. Immunol. 164:4375-4381.

13. Gombert, J.M., et al. 1996. Early quantitative and functional deficiency of NK1+-like thymocytes in the NOD mouse. Eur. I. Immunol. 26:2989-2998.

14. Baxter, A.G., Kinder, S.J., Hammond, K.J., Scollay, R., and Godfrey, D.I. 1997. An association between abTCR + CD4-CD8- T cell deficiency and IDDM in NOD/Lt mice. Diabetes. 46:572-582.

15. Mieza, M.A., et al. 1996. Selective reduction of V alpha $14+\mathrm{NK} T$ cells associated with disease development in autoimmune-prone mice. $J$. Immunol. 156:4035-4040.

16. Hammond, K.J.L., et al. 1998. Alpha/beta-T cell receptor (TCR)+CD4CD8- (NKT) thymocytes prevent insulin-dependent diabetes mellitus in nonobese diabetic (NOD)/Lt mice by the influence of interleukin (IL)-4 and/or IL-10. J. Exp. Med. 187:1047-1056.

17. Lehuen, A., et al. 1998. Overexpression of natural killer T cells protects Valpha14-Jalpha281 transgenic nonobese diabetic mice against diabetes. J. Exp. Med. 188:1831-1839.
18. Shi, F.D., et al. 2001. Germ line deletion of the CD1 locus exacerbates diabetes in the NOD mouse. Proc. Natl. Acad. Sci. USA. 98:6777-6782.

19. Wang, B., Geng, Y.B., and Wang, C.R. 2001. CD1-restricted NK T cells protect nonobese diabetic mice from developing diabetes. J. Exp. Med. 194:313-320.

20. Sharif, S., et al. 2001. Activation of natural killer T cells by alpha-galactosylceramide treatment prevents the onset and recurrence of autoimmune type 1 diabetes. Nat. Med. 7:1057-1062.

21. Hong, S., et al. 2001. The natural killer T-cell ligand alpha-galactosylceramide prevents autoimmune diabetes in non-obese diabetic mice. Nat Med. 7:1052-1056.

22. Wilson, S.B., et al. 2000. Multiple differences in gene expression in regulatory Valpha 24Jalpha Q T cells from identical twins discordant for type I diabetes. Proc. Natl. Acad. Sci. USA. 97:7411-7416.

23. Lee, P.T., Benlagha, K., Teyton, L., and Bendelac, A. 2002. Distinct functional lineages of human Valpha24 natural killer T cells. J. Exp. Med. 195:637-641.

24. Gumperz, J.E., Miyake, S., Yamamura, T., and Brenner, M.B. 2002. Functionally distinct subsets of CD1d-restricted natural killer T cells revealed by CD1d tetramer staining. J. Exp. Med. 195:625-636.

25. Gottlieb, P.A., and Eisenbarth, G.S. 1998. Diagnosis and treatment of pre-insulin dependent diabetes. Annu. Rev. Med. 49:391-405.

26. Benlagha, K., Weiss, A., Beavis, A., Teyton, L., and Bendelac, A. 2000. In vivo identification of glycolipid antigen specific T cells using fluorescent CD1d tetramers. J. Exp. Med. 191:1895-1903.

27. Benlagha, K., and Bendelac, A. 2000. CD1d-restricted mouse V $\alpha 14$ and human V $224 \mathrm{~T}$ cells: lymphocytes of innate immunity. Semin. Immunol. 12:537-542.

28. Bendelac, A., Bonneville, M., and Kearney, J.F. 2001. Autoreactivity by design: innate B and T lymphocytes. Nat. Rev. Immunol. 1:177-186.

29. Brossay, L., et al. 1998. CD1d-mediated recognition of an alpha-galactosylceramide by natural killer $\mathrm{T}$ cells is highly conserved through mammalian evolution. J. Exp. Med. 188:1521-1528.

30. Davodeau, F., et al. 1997. Close phenotypic and functional similarities between human and murine alphabeta T cells expressing invariant TCR alpha-chains. J. Immunol. 158:5603-5611.

31. Prussin, C., and Foster, B. 1997. TCR V alpha 24 and V beta 11 coexpression defines a human NK1 T cell analog containing a unique Th0 subpopulation. J. Immunol. 159:5862-5870. 